\title{
Chapter 2 \\ Disaggregated Hispanic Groups and Cancer: Importance, Methodology, and Current Knowledge
}

\author{
Paulo S. Pinheiro, Karen E. Callahan, and Erin N. Kobetz
}

\section{Introduction}

Hispanics living in the United States are heterogeneous: US-born and foreign-born; Mexican, Puerto Rican, Cuban, Dominican, Central American, South American, and a small number from Spain; wealthy and impoverished; English and/or Spanish speaking; residing in the North, South, East, or West; situated in cities or rural areas. These varying socioeconomic circumstances, nativity and/or immigration experiences, and cultural values and practices are strongly associated with cancer risk factors and thus impact cancer outcomes.

\section{Epidemiology of Cancer in Hispanics: Aggregated}

Cancer is the leading cause of death of all Hispanics combined [1], with the annual number of new cases diagnosed in 2014 exceeding 128,000 [2]. To address the increasing cancer burden of the burgeoning Hispanic population, including the

\footnotetext{
P. S. Pinheiro $(\square)$

Sylvester Comprehensive Cancer Center, University of Miami School of Medicine, Miami, FL, USA

Department of Public Health Sciences, University of Miami School of Medicine, Miami, FL, USA

e-mail: ppinheiro@med.miami.edu

K. E. Callahan

School of Community Health Sciences, University of Nevada Las Vegas,

Las Vegas, NV, USA

E. N. Kobetz

Sylvester Comprehensive Cancer Center, University of Miami School of Medicine, Miami, FL, USA
} 
development of cancer prevention and control strategies, all stakeholders, from clinicians to researchers to policymakers, must have timely and accurate populationbased cancer indicators, namely incidence, survival, and mortality. Incidence patterns are routinely reported by the North American Association of Central Cancer Registries (NAACCR) in their Cancer in North American (CiNA) Annual Reports [3] and the Annual Reports on Cancer, which group data from the Surveillance, Epidemiology, and End Results Program (SEER) and the National Program for Cancer Registries (NPCR) [4]. In addition, SEER provides data available for survival estimates of Hispanics [5], and mortality data are provided by the National Center for Health Statistics within the Centers for Disease Control and Prevention (CDC) [6].

However, accurate indicators for Hispanics are affected by specific problems. Hispanics are known to be undercounted at the cancer registry level $[7,8]$, largely due to incompleteness of the ethnicity variable. NAACCR protocols for the calculation of incidence rates reduce this undercount by using the NAACCR Hispanic Identification Algorithm (NHIA) which relies partly on Hispanic surname [9]. Mortality data also suffer from some degree of undercount, potentially up to 5\% [10]; however, routine use of similar algorithms does not take place. Nonetheless, Hispanic incidence and mortality estimates are generally consistent with each other and show that US Hispanics in aggregate have lower cancer incidence and mortality rates (overall and for the most common cancers) than the non-Hispanic white (NHW) referent group [1]. Important and well-known exceptions to this pattern are infection-related cancers, in particular cervix, liver, and stomach, for which Hispanics, examined in aggregate, have shown consistently higher rates than NHWs [1]. For survival, follow-up for foreign-born in general and Hispanics in particular can be difficult to perform, especially in comparison to NHWs and non-Hispanic Blacks [11]. However, SEER registries show that for all stages combined, cancer survival of Hispanics is similar or only slightly lower than NHWs, depending on cancer site [5].

Given the lower incidence, lower mortality, and relatively comparable survival among Hispanics in relation to NHWs, cancer has been frequently cited as another example of the "Hispanic Paradox," whereby Hispanics have positive health outcomes despite documented challenges with lower socioeconomic status and access to quality health care [12]. Another often cited positive characteristic in the study of health outcomes for Hispanics is the Healthy Immigrant Effect [13] (whereby immigrants are healthier on average than both their counterparts at home and the populations in the host countries) as a sizable portion of Hispanics are immigrants to the United States. The reality, however, may well be more complicated. Evidence from Fenelon et al. [14] has shown that the "Paradox" in terms of cancer mortality (and thus incidence) may largely be tobacco-related, and analyses of the potential survival parity or advantage for Hispanics on a population basis may be in part artifactual, a problem addressed later in this manuscript [11,15-17]. Additionally, cancer patterns among US-born Hispanics in relation to NHWs are not nearly as favorable as their foreign-born counterparts in the United States [18], offering a persuasive counterargument to the contention that any advantage stems from being 
Hispanic per se; rather, the advantage is at least partly being foreign-born, an advantage not exclusive to Hispanics, but also present among foreign-born Asians and Blacks [19, 20].

Such complexities are challenging, justifying the need for a distinct focus on cancer among Hispanics. Hispanics are demographically young, considerably heterogeneous, and, by and large, recent arrivals, as shown by the sevenfold increase in the Mexican Hispanic population between 1970 and 2010 [21]. Thus, they differ from the more established NHW and non-Hispanic Black populations and need a more critical and refined evaluation of their cancer indicators for a full understanding of their epidemiological patterns. Simply stated, aggregate estimates for all Hispanics that do not consider birthplace and the distinct Hispanic groups are masking considerable variation, with poor cancer outcomes seen among some segments of the Hispanic population that deserve additional targeted efforts to reduce disparities. Equally compelling is that careful examination of the determinants of these vast differences, whether risk or protective factors, can provide crucial information needed for effective public health and clinical intervention. In addition, any existing survival advantages among Hispanics could be hypothesis-generating and/or provide insights into improving cancer outcomes for other populations. Lastly, we demonstrate that specific Hispanic group analyses can provide new insights into the etiology of some cancers, insights only revealed by examining patterns among distinct Hispanic groups.

\section{Epidemiology of Cancer in Disaggregated Hispanic Groups}

\section{Challenges in the Data}

Cancer Registry Data (Incidence and Survival) Given their heterogeneity, much can be learned from examining the unique cancer profiles of all sizable Hispanic groups in the United States. These can be divided into 35 million Mexicans, 5.5 million Central Americans, 5.3 million Puerto Ricans, 4.1 million South Americans, 2.1 million Cubans, and 1.8 million Dominicans as of 2015 [22]. Additionally, examining differences between US-born and foreign-born (FB) groups adds clarity to the profiles, albeit only feasible with Mexican and Puerto Rican populations (for whom birth on the island is often analyzed as equivalent to FB), because of the sparsity of US-born cancer cases among the other Hispanic groups. NAACCR standards currently include the following specific categories of Hispanic ethnicity (group) for cancer cases: Mexican, Puerto Rican, Cuban, Dominican, and a single category for Central/South American, a convenient aggregation of very diffuse populations, despite having sociodemographic characteristics that are substantially different between the two. In addition, there are categories for other specified Hispanics including those from Spain, Hispanics by surname only, and Hispanics not otherwise specified (NOS) [3]. 
Overall, population-based analyses of cancer outcomes by Hispanic group and birthplace are obviously dependent on the completeness of these two data pieces. Unfortunately, both variables are substantially incomplete in cancer surveillance data required for incidence and survival statistics. In the most recent CiNA report released by NAACCR in 2017 [3], known specific group for 2010-2014 among Hispanic cancer cases was as low as 32\% in Texas and only 58\% in New York and New Jersey, all states with high Hispanic populations. Birthplace was only $50 \%$ complete for registry data in California (CA) [23] and 43\% in Florida (FL) [24], ideal states for studying differences between US-born and FB Mexican Hispanics (CA) and specific Hispanic groups (FL).

Mostly because the underlying data is complex and because of this considerable incompleteness, incidence rates of Hispanic groups have been estimated only a few times. Some researchers assigned groups ecologically at the county level, for example, Puerto Rican for all Hispanic cases residing in counties in New York City, Mexican for all Hispanics in Los Angeles County, and Cuban for all Hispanics in Miami-Dade and Broward counties in Florida [25, 26]. However, these methods are subject to substantial misclassification of Hispanic group at the individual level, leading to inflated estimates for some groups and underestimates for others. Other researchers assessed heterogeneity in risk among Hispanic groups using proportional incidence ratios (PIRs) [27, 28]; however, because PIRs do not depict the actual incidence of disease and are highly dependent on the relative frequency weight of each cancer, they have the potential to be misleading. To date, the only incidence rates for Hispanic groups calculated with individual level data was determined based on three years of Florida data (1999-2001) and included imputation of $32 \%$ of Hispanic cases to specific groups based on county of residence, cancer site, age and sex [29]. The study found that Mexicans in Florida had low cancer risk for most cancers except liver, cervical, and stomach, while Cubans and Puerto Ricans shared higher cancer risk compared to other Hispanic groups and had rates of endometrial, prostate, and colorectal cancer similar to NHWs [29]. Cubans more closely resembled NHWs with lower cervical and stomach cancers than other Hispanics, yet they surpassed NHWs for colorectal cancer, while Puerto Rican males showed particularly high liver cancer rates [29]. Some of these relative patterns observed for incidence are similar to current mortality analyses [30], suggesting that the underlying risk factors for each of these populations have not substantially changed in the last decade and attesting to their persistence in the respective Hispanic populations. Since then, no other study has attempted to estimate population-based incidence rates for Hispanic groups.

Like incidence research, and owing to many of the same shortcomings, few studies have analyzed differential cancer survival by Hispanic group. These projects were mostly conducted in Florida [15,31], the state with sufficient heterogeneity and numerically sizeable Hispanic groups to conduct such studies, but were subject to some important biases, as discussed in more detail below. SEER provides survival statistics for Hispanics, which are predominantly reflective of the experience of Mexican Hispanics given the overwhelming proportion of this group in the SEER 
coverage area [5]. However, because of incompleteness of place of birth, SEER survival statistics reports do not make a distinction between US- and foreign-born Hispanics [5]. Overall, the consequence of this obstacle in the data and its consequences (see below) is a virtual dearth of knowledge regarding differences in survival among specific Hispanic groups and compared to other non-Hispanic populations.

Vital Statistics Data (Mortality) In contrast to surveillance data (used for incidence and survival), mortality data, particularly when obtained directly from states, can be assembled to achieve near completeness for Hispanic specific groups. Another advantage of mortality data is that with additional work using specific place of birth and text fields, it is possible to study Central Americans and South Americans separately, which is not possible in cancer registry data. Thus, mortality data are optimal to analyze Hispanic heterogeneity in detail.

While the National Vital Statistics System data from the CDC [6] have the advantage of covering the entire nation, the available data lack sufficient detail on some key variables, such as specific country of birth and ethnicity text fields. These federal datasets are compiled from each state's data and rely on broader variables (e.g., US versus foreign birthplace, South/Central American ethnicity) which inevitably leads to some degree of misclassification [6]. As an example, for cancer deaths that occurred between 2010 and 2016 in the diverse state of Florida, 29\%, 24\%, 17\%, $10 \%$, and $9 \%$ of individuals born in Paraguay, Spain, Argentina, Venezuela, and Honduras, respectively, were coded as non-Hispanic [32]. Furthermore, of all Argentinians categorized as Hispanic, coded Argentina by birthplace and/or text fields, $38 \%$ were not correctly categorized in the South/Central American category, most likely falling into the Ethnicity Other category. The pattern continued with at least 20\% of those who were known to be from Central/South America (Nicaraguans, Colombians, etc.) and not found in the South/Central American grouping category [32]. On the other hand, individuals born in countries such as Brazil, Italy, and Portugal have substantial proportions recorded as Hispanic ethnicity, when this does not correlate with the US Office of Management and Budget (OMB) definition of Hispanic [33]. While this misclassification results in some underestimation of Hispanics as a whole, its final effect on estimated rates is more pronounced when studying specific Hispanic groups, with misclassification across groups as well as a variable proportion of Not Otherwise Specified Hispanic cases (NOS).

However, at the state level, mortality data for some states contains the necessary detailed information that allows for accurate specific group classification based on codes for ethnic groups and specific birthplace, augmented with revealing text descriptive for otherwise incompatible or incomplete cases. In our studies [18, 30, 34], we found data in three states (California, New York, and Florida) to be more than $97 \%$ complete with a traceable specific Hispanic group leaving only $3 \%$ of cases as Hispanic NOS. Notably, the availability of ethnicity group, birthplace, and text fields was partial for other states (Texas) and unavailable for Maryland and New Jersey, limiting their use for this purpose. 


\section{Challenges in the Analyses}

Primary to the accurate disaggregation of Hispanics into unique groups is the identification of all those in the population who are indeed Hispanic. While acknowledging that race and ethnicity are social constructs that vary by geography across the globe, within the United States, disparities research has followed the OMB-defined division [33] of races into four mutually exclusive categories (and mixed race) while ethnicity is coded as Hispanic or Latino, via a binary Yes/No. Once a case is coded Hispanic, then depending upon the data source and how it is collected, specific group allocation follows. One of the greatest challenges in population-based studies is how to best handle those persons who cannot be allocated definitively to a group, commonly referred to as Not Otherwise Specified, or Hispanic NOS. Importantly, these persons do not constitute an actual distinct group, as each case logically belongs to a specific group (or combination of groups) at some point whether in the present or by heritage from past generations. Despite that, a minority of cases (mostly US-born) self-identify as Hispanic only. However, a comparison of incidence data (low completeness of Hispanic specified group) to mortality data (very high completeness of Hispanic specified group) shows that more often, specific group information is known to the individual case, but is not asked, not known, or not available to those who record the data. Thus, assignment to the Hispanic NOS category is commonly a result of incomplete information. How researchers attend to these cases determines the accuracy of resulting calculated indicators.

\section{Incidence and Mortality Rate Problems for Disaggregated Hispanic}

Groups Management of Hispanic NOS cases varies between the sources of data used for calculating cancer outcomes. Cancer registry data for cancer incidence and death data for mortality rates are used in the numerators, and census-based population data are used in the denominators. Census reports have traditionally grouped Hispanics into five categories, with only three corresponding to specific groups: Mexicans, Puerto Ricans, and Cubans. Other Specified Hispanics (which includes any specified group such as Dominican, Central American, South American, Spaniard, etc.) make up a fourth group, and a very sizeable Hispanic NOS group is the last. Thus, Hispanic NOS cases in the numerator, whether derived from incomplete incidence data or more complete mortality data, do not correspond to the NOS cases in the denominator, which raises a critical compatibility issue. When estimating rates for these specific groups, the lack of proper handling of NOS cases with the correction of this imbalance can truly confound our understanding of patterns among Hispanics.

Mortality rates are a good example. Without attending to the fact that the total number of NOS cases in census data logically includes Hispanics from potentially all groups (including Mexican, Puerto Rican, and Cuban) [35], people will be missing from the denominator for the three groups that are specified: Mexican, Puerto Rican, and Cuban. Another common problem arises in studies which combine the fourth (Other Specified, i.e., South and Central Americans, Dominicans) and fifth 
census groups (Hispanic NOS) into one denominator group and combine all nonMexican, non-Puerto Rican, and non-Cuban cancer cases into one numerator group. These NOS mismatches between numerators and denominators result in an overestimation of death rates for the Mexican, Puerto Rican, and Cuban groups, with a meaningless underestimation of the Hispanic NOS mortality rates (often labeled as Other Hispanics or South and Central American, more for convenience than accuracy). For incidence rates, the problem is even worse because the specific group information in the numerator is substantially less complete than mortality data; and without accounting for the Hispanic NOS, these group-specific rates are inevitably underestimated. Sadly, some research published in reputable journals fail to meet the basic logical tenet that the sum of the parts should equals the whole; rather these studies present disaggregated results where the sum of the weighted rates for each group does not correspond to the total All Hispanics rate. Errors of this nature arise from the complexity of managing the Hispanic NOS cases; treating NOS cases as an included unique group and excluding them are both problematic. Thus, as in our research, exhaustive ascertainment of specific group in both the cancer data and the denominator data from available detailed sources [22], followed by treatment of the remaining (hopefully minimal) NOS cases via appropriate partition and/or imputation strategies, is essential for the presentation of the true cancer incidence and mortality rates in Hispanic groups. In addition, the partition and/or imputation should always take into account birthplace distributions [36] in both the cancer data and the population data.

\section{Survival Estimation Problems for Disaggregated Hispanic Groups}

Hispanic NOS Survival estimates on a population level are derived from one data source, cancer registries, and thus theoretically should avoid the problem of numerator/denominator mismatch. Survival denominators are all the cancer cases, while numerators are those who have survived up until a certain designated period of time. However, a crucially important methodological barrier to calculate accurate survival for groups also includes the "nebulous" Hispanic NOS category and who it represents. In cancer surveillance data (registries), a proper specific group is more likely specified when the death has occurred because extra information on ethnicity and/ or birthplace is available from direct access to and/or linkages with death certificate data. Consequently, having a specified Hispanic group in registry data is positively correlated with death, the exact outcome of interest in survival analysis [16, 17]. Conversely, if a Hispanic case is not deceased, the information available for precise group and birthplace is much less available, making it more likely to be ascribed as Hispanic NOS, rather than Mexican, Cuban, etc. The resulting survival estimates for the specified Hispanic groups will thus be underestimated, or lower than reality, precisely because those who are alive from any given specific group are more likely 
to be coded NOS while those who are dead are more likely to have a specified group $[16,17]$. In practical terms, this results in a specific group likely appearing to have worse (lower) survival than reality, while the Hispanic NOS survival will have better (higher) survival than that seen for all Hispanics combined, as these NOS cases are more likely to appear alive $[16,17]$. Thus, exclusion of Hispanic NOS cases in survival analysis, under the false assumption that membership in the NOS group is random, results in highly biased results, with a specified group having biased low survival compared to other non-Hispanic groups (e.g., NHWs), since some alive cases are being excluded. Additionally, even if Hispanic NOS cases are excluded and analysis is restricted to Hispanic known groups only, survival comparisons will still be biased. This is because Puerto Ricans and Cubans are two groups for which death matches are more complete than Mexicans and Central and South Americans, given the higher proportion among Puerto Ricans and Cubans of a workable social security number, the essential variable for death linkages [11,15]. As it stands, on a population basis, exclusion of NOS cases misses the population-based characteristics of the data for each of the Hispanic groups and results in making their outcomes look worse than reality.

Disproportionate Loss to Follow-up Survival studies have identified artifactual factors impacting death linkages to cancer registry data, particularly for minority groups with substantial proportions that are foreign-born such as Hispanics [11, 15]. Linkage problems arise from an inability to match social security numbers (SSN) because of diverse reasons including lack of SSNs, incorrect SSNs resulting in nonmatches, or different structure of surnames or misspellings, common among Hispanics as well as among Asians from countries with non-Roman alphabets [11]. Missed deaths also arise from cases that are diagnosed in the United States but die in another country. These may be non-residents who are falsely coded as resident when coming to the United States solely for diagnosis and treatment, often referred to as "medical tourism." Others may be residents who are diagnosed here but return to their home countries to die, a phenomenon referred to as the "Salmon Bias" [37].

In all the described scenarios, persons from minority populations, especially those with late stage disease, are disproportionately lost to follow-up [11]. This may bias their survival upward (better than reality), especially in non-SEER states with registries associated with the CDC's National Program of Cancer Registries (NPCR). The difference between the registry types arises because SEER registries benefit from the requirement of a minimum of $95 \%$ of cases having a precise date of alive contact over time [38]. Unfortunately, this requirement is not specific to ethnicity or birthplace; thus, the remaining 5\% are alive/living cases, disproportionately foreign-born and minority cases. By accruing more accurate survival time, SEER substantially reduces the potential for bias from non-random censoring among the foreign-born. However, in NPCR registries, no date of last alive contact is recorded, and if a specific patient does not match any record in a mortality list at a given date, then that patient is presumed alive at that date, which is called the "presumed alive" assumption of survival [11]. The following example illustrates the 
current difference between SEER and NPCR survival data: Mrs. X is a US resident of Venezuelan origin who had lung cancer diagnosed in the United States. After 4 months of treatment in the United States, she returns to her birth country and subsequently passes away in Venezuela. The death data for Mrs. X never reaches mortality records in the United States. In SEER registries, her vital status would be counted as alive, but only for 4 months of survival time, based on her last medical encounter recorded in the United States. In NPCR registries, she would be presumed alive until the date of cut-off for the survival estimation, often 5 years. Thus, the NPCR registry would have substantially more months of survival for Venezuelans than the SEER registry. Similarly, having an unworkable SSN or a misspelled last name (both problems more prevalent among the foreign-born) would work the same way even if there was no return to Venezuela, because they could more likely result in a missed death after routine death linkages. Compared to other groups, deaths of Puerto Ricans and Cubans are more likely to be fully captured and linked in cancer data [15]. This is because Puerto Ricans are US citizens, and Cubans have lower likelihood of returning to Cuba and historically greater facility in acquiring legal status in the United States. While on a population basis the proportion of missed deaths is small, these missed deaths, particularly for poor prognosis cancers, in Hispanic groups that are largely foreign-born (especially Central and South Americans [15] and Mexicans born in Mexico), result in inflation of survival estimates, making comparisons inaccurate [11]. To make things even more confusing, the combined effect of these biases - Hispanic NOS and higher loss to follow-upcan send survival estimates in any direction away from reality depending on which one is stronger or just balance each other out.

\section{Current Knowledge: Cancer in Hispanic Groups, Based on Mortality Data}

Using mortality data, we conducted research that either addressed or bypassed the common deficiencies in the literature for disaggregated Hispanic groups. We used death certificate data and carefully assembled multiple race/ethnicity fields, text fields, and available birthplace variables to accurately classify Hispanic group. Here we summarize our findings from population-based studies using disaggregated Hispanic groups, highlighting four examples to show the importance of disaggregation.

\footnotetext{
Mexicans and Puerto Ricans in the United States Compared to Those in Mexico and Puerto Rico Mexicans and Puerto Ricans are the largest US Hispanic groups traceable to a single country of origin. (The island of Puerto Rico, although an American territory, is considered here as a country of origin for convenience.) Using methodology from other studies, we compared cancer rates for these specific groups in the states where they are most populous, California for Mexicans and New York for Puerto Ricans, to cancer rates among their counterparts in Mexico and Puerto Rico, respectively (Table 2.1) [34, 39].
} 
For the vast majority of cancers, mortality during the studied time period (20082012 in California and 2008-2014 in New York) was higher in the Hispanic groups residing in the United States than in their countries of origin [40, 41] (Table 2.1). Given that cancer survival is higher in the continental United States than in Mexico and Puerto Rico [42], higher mortality suggests that cancer incidence must be substantially higher in the United States for the majority of cancers. Differences in risk among genetically and culturally similar populations such as these are commonly attributed to a higher prevalence of lifestyle risk factors in the United States, including smoking, obesity, alcohol, and other substance abuse [43]. Thus, results showing higher mortality in the United States are not surprising for many cancers including lung and other tobacco-related cancers; breast cancer, linked to obesity but also likely to differences in fertility patterns; liver cancer, linked to obesity and chronic infection with the hepatitis $\mathrm{C}$ virus (HCV); and other obesity-related cancers such as kidney and endometrial. The significantly higher pancreas and nonHodgkin lymphoma mortality patterns are more intriguing, given how little etiological knowledge is known for these cancers that could be explained logically on a population basis. For a few cancers, mortality rates are lower in the United States among the Mexican and Puerto Rican groups compared to their country-oforigin counterparts. These include prostate cancer, an interesting pattern seen in other migration studies [20,39,44] and likely reflective of better survival in the United States including more aggressive treatment in older ages and more access to varied and complex treatment regimens, notwithstanding the potential effect of the comparatively higher PSA screening patterns in the United States.

Interesting patterns that emerge between these two distinct Hispanic groups include Mexican American women seeming to escape the adverse HCV impact, with lower liver cancer rates than women in Mexico. Also, excesses in colorectal cancer for Mexican American men compared to Mexico are not mirrored in Puerto Rican patterns. Conversely, the gains seen among Mexican Americans for cervical and stomach cancers are not realized among Puerto Ricans in New York, who share similar high mortality with their island counterparts (Table 2.1). Some findings from similar analyses using available data from US minorities and their countries of origin extend to childhood cancer: for instance, Mexican American children have higher rates (incidence and mortality) of brain cancer and neuroblastoma compared to Mexican children in Mexico [39], which raises provocative questions about an increased risk for these cancers in the US environment. Yet, very few of these questions have received attention from the research community.

\section{Differential Cancer Patterns over the Lifespan (Based on Birthplace) among} Hispanics in the United States Of the 35 million Hispanics in the US Mexican group, 22 million are US-born and 13 million are foreign-born [45]. Leveraging this distribution in analyses disaggregated by birthplace provides additional insights into the role of environmental factors in the development of cancers. While birthplace is by no means a perfect indicator of residential history or indeed any risk factors for cancer, on a population basis, it is the most complete proxy of early life environmental influences, as most immigrants arrive as adults [45]. Using mortality data from California and Texas, we showed that cancer patterns of Hispanics vastly 


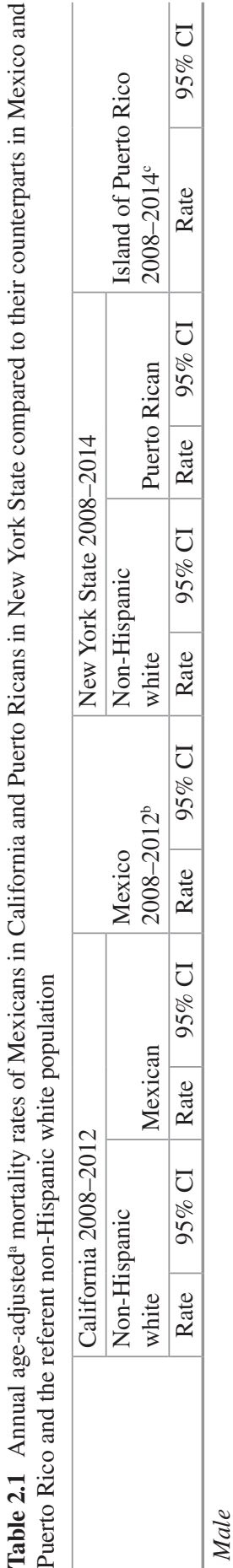

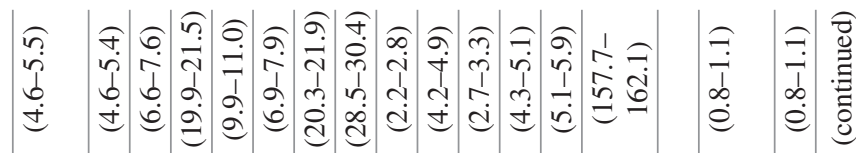

尚

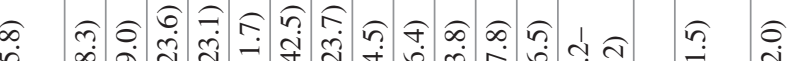

i

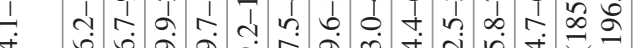

巳

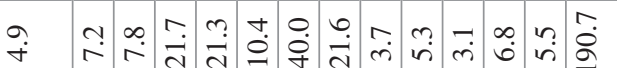

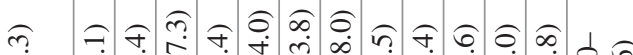

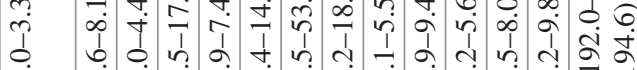

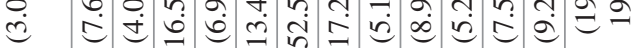

c)

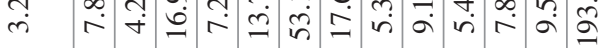

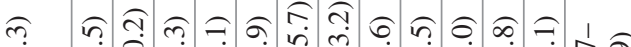

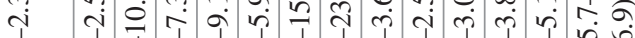

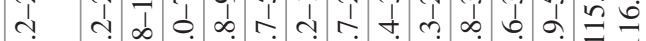

a d

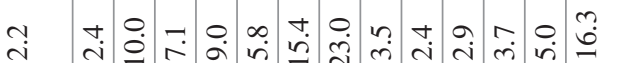

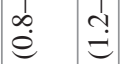

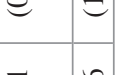

$\exists \quad \stackrel{0}{-}$

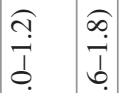

$\stackrel{0}{=} \stackrel{0}{=}$

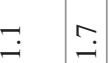

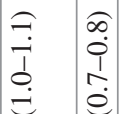

$\stackrel{e}{\ominus}$

-

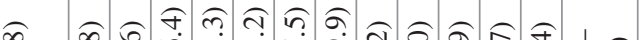

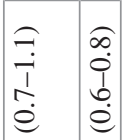

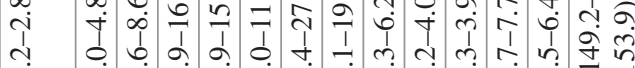

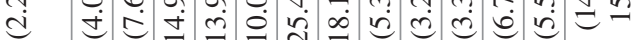

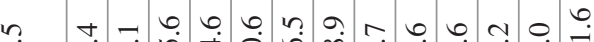

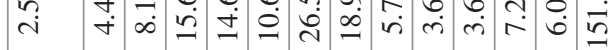

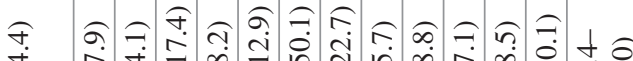

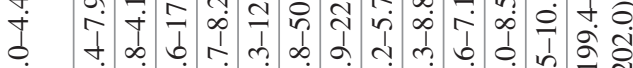

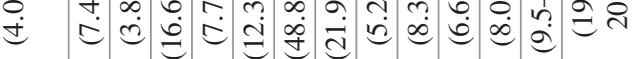

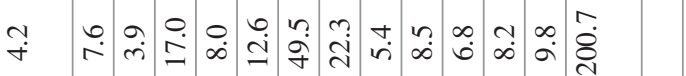

o.

00

Iิ

ㄹ.

○.

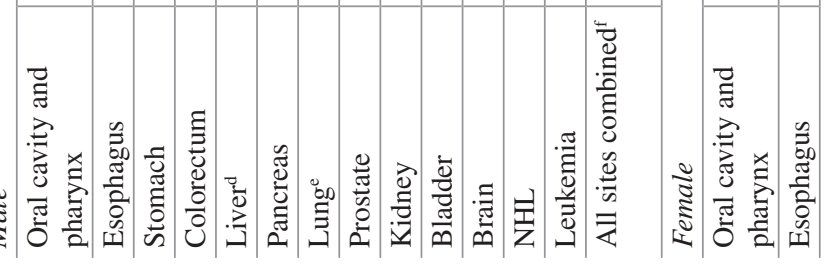




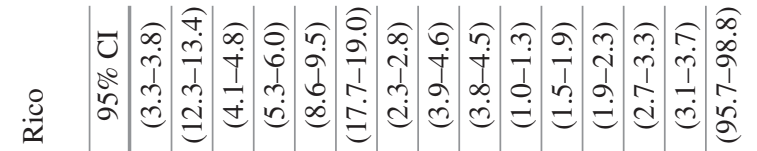

$\stackrel{\circ}{\frac{0}{2}}$

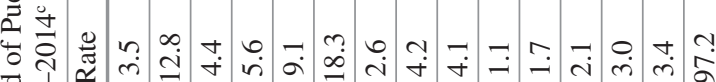
葛

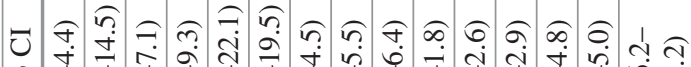

चี .0

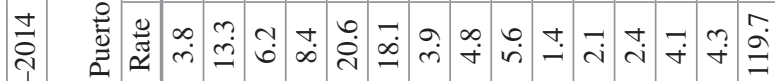

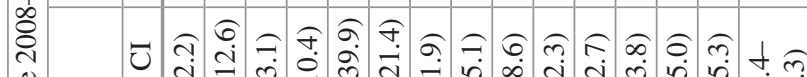

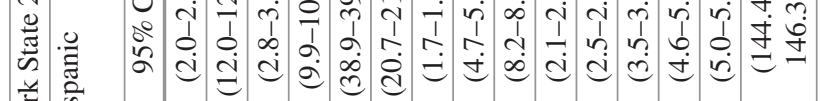

竞

ż

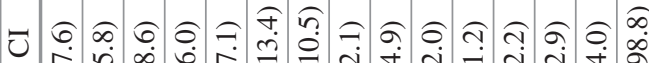

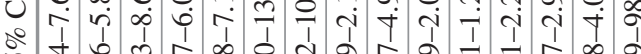

స̆丶 유

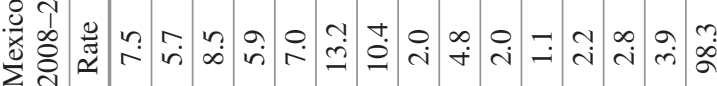

ઇ તุ

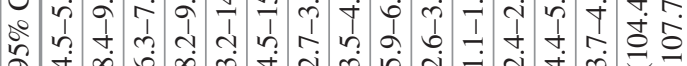

:

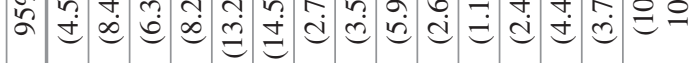

苂

政

ำ

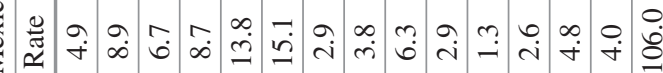

๑)

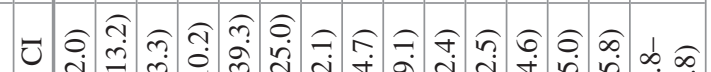

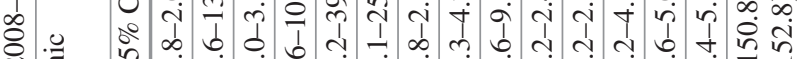

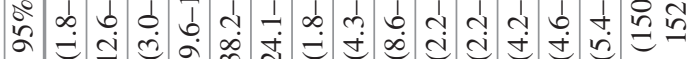

N

(8)

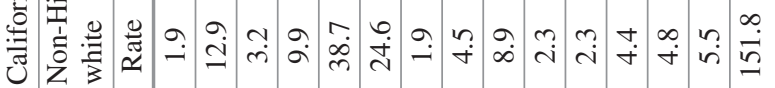

$\overrightarrow{\mathrm{i}}$

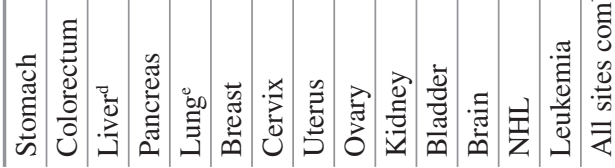

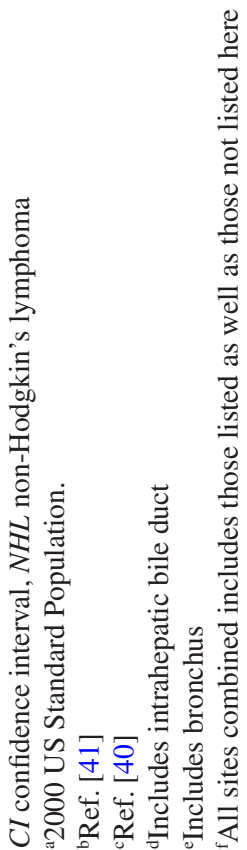


differ by birthplace [18]. Contrary to the purported Hispanic advantage portrayed when rates are calculated in aggregate, overall cancer mortality rates for US-born Hispanic males are for the most part no better than non-Hispanic whites, albeit with site-specific variation including lower rates of tobacco-related cancers such as lung and bladder cancers and higher rates of liver, stomach, colorectal, and kidney cancers [18]. Conversely, foreign-born Hispanics are a relatively low-risk group, burdened only with higher rates of stomach, cervix, and prostate cancers than their US-born counterparts [18], a finding further confirmed when analyzing Mexican Hispanic populations alone [39]. Most striking are the excesses seen for male colorectal, kidney, and liver cancers, likely reflecting higher prevalence of obesity and $\mathrm{HCV}$ infection among US-born Mexicans than their foreign-born counterparts. Barring ascertainment of detailed risk factor information, virtually impossible at the individual-level on a population basis, these patterns may be the best evidence we have so far of the impact of spending formative years in a US environment characterized by overconsumption of fats and sugars, sedentary lifestyle, and low age of experimentation with drugs and alcohol as well as unique stressors associated with discrimination [43]. Moreover, disaggregated rates by birthplace provide opportunities to identify protective factors seen in foreign-born populations, identify strategies to maintain these protective factors, and potentially use this information in risk reduction strategies among US-born populations.

\section{Puerto Ricans and Divergence from Favorable Aggregated Hispanic}

Patterns Using mortality data from New York State (NYS) [34], we revealed considerable cancer mortality disparities for Puerto Ricans compared both to the majority NHW population and to other Hispanic groups [34]. These disparities, which should be addressed by targeted cancer prevention and control programs, were largely masked by the presentation of Hispanic cancer mortality rates in aggregate.

Puerto Ricans are the largest Hispanic group in NYS [22]; moreover, most cancer deaths $(81 \%)$ in this group occurred among Puerto Ricans born in the continental United States [34]. Thus, examination of this group in this state provides a unique lens with which to examine the influence of extended acculturation as a minority in the United States. Three prominent findings emerged from our study. First, similar to US-born Hispanic males in California and Texas, overall cancer mortality rates of Puerto Rican males in NYS are not lower than, but rather similar to rates of the majority NHW reference group. Again, different cancer sites afflict the two populations: tobacco-related bladder and lung cancer mortality are higher among NHWs; infection-related cancers (stomach, liver and cervix) are higher among Puerto Ricans [34]. Secondly, the disparity between Puerto Ricans and NHWs for these infection-related cancers, more commonly associated with developing countries, is consistent with patterns seen for another socio-economically deprived US minority: the US-born black population [34]. Prior assumptions that the high burden among Hispanics in aggregate for infection-related cancers was being driven by the foreignborn (carrying their risk from developing countries) should be urgently re-examined. This evidence from disaggregation of rates suggests US poverty, proportionately 
higher in NYS among Puerto Rican and US-born blacks, is driving the burden, and thus appropriate resources should be shifted to eliminate this disparity. Lastly, Puerto Rican males in NYS have significantly higher mortality than any other Hispanic group for oral, esophageal, colorectal, lung, bladder, and especially liver cancer, while Puerto Rican females have higher mortality from colorectal, lung, postmenopausal breast, liver, and cervix cancers [34]. With the exception of colorectal and breast cancers, these are the cancers associated with low SES [46]. Thus, these patterns, revealed only because of disaggregation, not only are consonant with the negative effects often associated with acculturation, but also correlate with prevailing economic disparities that adversely impact health possibly more acutely in second-generation immigrants [34].

\section{Liver Cancer: Etiological Insights from Disaggregation of Hispanic Groups Our}

fourth and final example illustrating the importance of disaggregation focuses specifically on liver cancer, drawing again from our study conducted in NYS [34]. As a multi-causal disease [47], liver cancer incidence and mortality are difficult to interpret given disparate etiological factors, including HCV, obesity, heavy alcohol consumption, diabetes, and chronic infection with the hepatitis B virus, the latter especially among Asians and the foreign-born [48]. We examined Hispanics as a whole and disaggregated: Puerto Rican male liver cancer rates were higher than other analyzed groups in NYS, including Asians and blacks [34]. Within the 19451965 birth cohort, known for its high prevalence of HCV infection [49], excesses in liver cancer mortality were exceedingly high among not only male but also female Puerto Ricans. Patterns were similar to US-born blacks, but diverged completely from other Hispanic groups in New York, which include overwhelmingly foreignborn Hispanics (Dominicans, Central Americans, South Americans, etc.) as well as non-Hispanic blacks born in the Caribbean; all showing relatively low mortality for liver cancer [34]. Differences are more pronounced in males and within the birth cohort of 1945-1965, suggesting just from a descriptive analysis that socially and economically deprived minority populations physically present in NYS during the 1960s-1980s (when transmission of HCV was likely caused by IV drug use and needle sharing) who also share disproportionately high rates of incarceration [50], linked to HCV transmission [51], are particularly prone to higher mortality from liver cancer. While undeniably other etiological factors contribute to the excess of liver cancer among Hispanics in general, especially in older populations, the existing patterns suggest a compelling association between the known high prevalence of HCV [52] and liver cancer [34] in the Puerto Rican population, even in relation to NHWs within the same birth cohort, who are also considered high-risk. Notably, neither Hispanics overall nor Puerto Ricans specifically are currently considered a priority population for viral hepatitis control programs [53]; yet, our finding, which would have been totally missed had it not been for the disaggregation of Hispanics, suggests that HCV testing and viral hepatitis control could help alleviate this disparity particularly for Puerto Ricans. Further study of the etiology of liver cancer by disaggregated groups will provide additional clarification into the specific etiological forces driving high cancer rates among other Hispanic groups, particularly in older populations. 


\section{Conclusions}

US Hispanics number 55 million, which is $17 \%$ of the total US population [22]; they are highly heterogeneous, with unique genetic admixtures and widely variant socioeconomic profiles $[45,46]$. While the population structure of Hispanics is relatively young, these currently younger Hispanics will soon reach the ages at which cancer is more common. Prevention strategies are needed now to meet this need. Special attention should be directed to the distinct disparities among US-born Hispanics, whose cancer numbers are rapidly trending upwards and who may not profit from the same protective health benefits of their first-generation immigrant counterparts. Accurate incidence, survival, and mortality rates of Hispanics by specific group are critical because aggregation provides at best a fuzzy picture and at worst a lie. Not all Hispanics are doing well, as would be suggested by aggregated rates. There are many challenges to studying population-based cancer indicators by disaggregated Hispanic groups, especially for incidence and survival, which we described above. Yet, overcoming these challenges can provide critical insights, as we demonstrated here through a synthesis of our results from several recent studies.

While cancer surveillance and vital statistics data have the advantage of being available on an individual level for entire populations, they are limited to basic demographic information. Certainly, while our findings are hypothesis-generating, further research that incorporates individual-level risk factor information will be required to substantiate and further explain these findings for Hispanic groups, including prevalence of obesity, smoking, diabetes, age at immigration, and length of time residing in the United States (for immigrants). Studies should also examine other social, economic, and cultural factors that impact access to health care and attitudes toward health, which may differ substantially by specific Hispanic group. Moreover, finding answers to the innumerous questions arising from these comparisons will require the inclusion of biological characteristics of tumors, including genetic and molecular subtypes.

Continued epidemiological research on the intra-ethnic cancer experience among Hispanics in the United States is imperative, not only to identify and address disparities, but also because this highly heterogeneous population provides opportunities through specific group analyses to further explore the etiology of cancers and discover potential avenues for cancer prevention and control efforts.

\section{References}

1. Siegel RL, Fedewa SA, Miller KD, Goding-Sauer A, Pinheiro PS, Martinez-Tyson D, et al. Cancer statistics for Hispanics/Latinos, 2015. CA Cancer J Clin. 2015;65(6):457-80.

2. United States Cancer Statistics: 1999-2014 incidence, WONDER Online Database. United States Department of Health and Human Services, Centers for Disease Control and Prevention and National Cancer Institute; 2016. http://wonder.cdc.gov/cancer-v2014.html. Accessed 29 Apr 2018. 
3. Copeland G, Lake A, Firth R, Wohler B, Wu XC, Schymura M, De P, Hofferkamp J, Sherman R, Kohler B, editors. Cancer in North America: 2010-2014. Volume One: Combined cancer incidence for the United States, Canada and North America. Springfield: North American Association of Central Cancer Registries, Inc.; 2017.

4. Jemal A, Ward EM, Johnson CJ, Cronin KA, Ma J, Ryerson AB, et al. Annual report to the nation on the status of cancer, 1975-2014, featuring survival. J Natl Cancer Inst. 2017;109(9).

5. Mariotto AB, Noone AM, Howlader N, Cho H, Keel GE, Garshell J, et al. Cancer survival: an overview of measures, uses, and interpretation. J Natl Cancer Inst Monogr. 2014;2014(49):145-86.

6. National Center for Health Statistics. NVSS—National Vital Statistics System. Ctr Dis Control Prev. https://www.cdc.gov/nchs/nvss/index.htm. Accessed 29 Mar 2018.

7. Gomez SL, Glaser SL. Misclassification of race/ethnicity in a population-based cancer registry (United States). Cancer Causes Control. 2006;17(6):771-81.

8. Pinheiro PS, Sherman R, Fleming LE, Gomez-Marin O, Huang Y, Lee DJ, et al. Validation of ethnicity in cancer data: which Hispanics are we misclassifying. J Registry Manag. 2009;36(2):42-6.

9. NAACCR Race and Ethnicity Work Group. NAACCR guideline for enhancing Hispanic/ Latino identification: revised NAACCR Hispanic/Latino identification algorithm [NHIA v2.2.1]. Springfield: North American Association of Central Cancer Registries; 2011.

10. Arias E, Schauman WS, Eschbach K, Sorlie PD, Backlund E. The validity of race and Hispanic origin reporting on death certificates in the United States. Vital Health Stat 2. 2008;(148):1-23.

11. Pinheiro PS, Morris CR, Liu L, Bungum TJ, Altekruse SF. The impact of follow-up type and missed deaths on population-based cancer survival studies for Hispanics and Asians. J Natl Cancer Inst Monogr. 2014;2014(49):210-7.

12. Markides KS, Coreil J. The health of Hispanics in the southwestern United States: an epidemiologic paradox. Public Health Rep. 1986;101(3):253-65.

13. Kennedy S, Kidd MP, McDonald JT, Biddle N. The healthy immigrant effect: patterns and evidence from four countries. J Int Migrat Integrat. 2015;16(2):317-32.

14. Fenelon A. Revisiting the Hispanic mortality advantage in the United States: the role of smoking. Soc Sci Med. 2013;82:1-9.

15. Pinheiro PS, Williams M, Miller EA, Easterday S, Moonie S, Trapido EJ. Cancer survival among Latinos and the Hispanic Paradox. Cancer Causes Control. 2011;22(4):553-61.

16. Pinheiro PS. The influence of Hispanic ethnicity on nonsmall cell lung cancer histology and patient survival. Cancer. 2013;119(6):1285-6.

17. Pinheiro PS, Bungum TJ. Country of origin and breast cancer survival. Asia Pac J Clin Oncol. 2014;10(3):279.

18. Pinheiro PS, Callahan KE, Gomez SL, Marcos-Gragera R, Cobb TR, Roca-Barcelo A, et al. High cancer mortality for US-born Latinos: evidence from California and Texas. BMC Cancer. 2017;17(1):478.

19. Jin H, Pinheiro PS, Xu J, Amei A. Cancer incidence among Asian American populations in the United States, 2009-2011. Int J Cancer. 2016;138(9):2136-45.

20. Pinheiro PS, Callahan KE, Ragin CR, Hage RW, Hylton T, Kobetz EN. Black heterogeneity in cancer mortality: US-Blacks, Haitians, and Jamaicans. Cancer Control. 2016;23(4):347-58.

21. Gutiérrez DG. A historic overview of Latino immigration and the demographic transformation of the United States. In: Gutierrez RA, Almaguer T, editors. The New Latino studies Reader: a twenty-first-century perspective. Oakland: University of California Press; 2016. p. 108-25.

22. Ruggles S, Genadek K, Goeken R, Grover J, Sobek M. Integrated public use Microdata Series: version 6.0 [machine-readable database]. American Community Survey 2015 1-year estimate. Minneapolis: University of Minnesota. Accessed Feb 2018.

23. California Cancer Registry (www.ccrcal.org), California Department of Public Health. SEER $*$ Stat Database: Incidence—California, Jan 2018 (1988-2015), 02/16/2018; NAACCR 3339 Version. Benchmarked 1988-1989 DOF population estimates, 6/12/2006; NCHS population estimates 1990-2015. 
24. Florida Cancer Data System, Bureau of Chronic Disease Prevention, Florida Department of Health, Tallahassee, FL. 2010-2014. https://fcds.med.miami.edu/inc/statistics.shtml. Accessed 31 Jan 2018.

25. Trapido EJ, Burciaga Valdez R, Obeso JL, Strickman-Stein N, Rotger A, Perez-Stable EJ. Epidemiology of cancer among Hispanics in the United States. J Natl Cancer Inst Monogr. 1995;(18):17-28.

26. Howe HL, Lake A, Schymura MJ, Edwards BK. Indirect method to estimate specific Hispanic group cancer rates. Cancer Causes Control. 2009;20(7):1215-26.

27. Howe HL, Wu X, Ries LA, Cokkinides V, Ahmed F, Jemal A, et al. Annual report to the nation on the status of cancer, 1975-2003, featuring cancer among US Hispanic/Latino populations. Cancer. 2006;107(8):1711-42.

28. Stern MC, Zhang J, Lee E, Deapen D, Liu L. Disparities in colorectal cancer incidence among Latino subpopulations in California defined by country of origin. Cancer Causes Control. 2016;27(2):147-55.

29. Pinheiro PS, Sherman RL, Trapido EJ, Fleming LE, Huang Y, Gomez-Marin O, et al. Cancer incidence in first generation U.S. Hispanics: Cubans, Mexicans, Puerto Ricans, and new Latinos. Cancer Epidemiol Biomarkers Prev. 2009;18(8):2162-9.

30. Pinheiro PS, Callahan KE, Siegel RL, Jin H, Morris CR, Trapido EJ, et al. Cancer mortality in Hispanic Ethnic Groups. Cancer Epidemiol Biomarkers Prev. 2017;26(3):376-82.

31. Chinea FM, Patel VN, Kwon D, Lamichhane N, Lopez C, Punnen S, et al. Ethnic heterogeneity and prostate cancer mortality in Hispanic/Latino men: a population-based study. Oncotarget. 2017;8(41):69709.

32. Bureau of Vital Statistics, Florida Department of Health, Jacksonville, Florida. Mortality data 2008-2012, received June 2015.

33. US Census Bureau. Population: Hispanic origin. Census.gov. https://www.census.gov/topics/ population/hispanic-origin/about.html. Accessed 31 Jan 2018.

34. Pinheiro PS, Callahan KE, Boscoe FP, Balise R, Cobb TR, Lee DJ, et al. Cancer-site-specific disparities in New York, including the 1945-1965 birth cohort's impact on liver cancer patterns. Cancer Epidemiol Biomarkers Prev. 2018;27(8):917-27. https://doi.org/10.1158/10559965.EPI-18-0194.

35. Cresce AR, Ramirez RR. Analysis of general Hispanic responses in census 2000. Population division working paper 2003;(72).

36. Pinheiro PS, Bungum TJ, Jin H. Limitations in the imputation strategy to handle missing nativity data in the Surveillance, Epidemiology, and End Results program. Cancer. 2014;120(20):3261-2.

37. Razum O. Commentary: of Salmon and time travellers-musing on the mystery of migrant mortality. Int J Epidemiol. 2006;35(4):919-21.

38. SEER Training Modules, Requirements of Commission on Cancer/Governing Agencies. U. S. National Institutes of Health, National Cancer Institute. https://training.seer.cancer.gov/followup/intro/requirements.html. Accessed 15 Mar 2018.

39. Pinheiro PS, Callahan KE, Stern MC, de Vries E. Migration from Mexico to the United States: a high-speed cancer transition. Int J Cancer. 2018;142(3):477-88.

40. United States and Puerto Rico Cancer Statistics, 1999-2014 mortality, WONDER Online Database. United States Department of Health and Human Services, Centers for Disease Control and Prevention. 2016. https://wonder.cdc.gov/controller/datarequest/D137; jsessioni d=13D799C81A1D2F665FB2846022B1B27B. Accessed 3 Apr 2018.

41. WHO Mortality Database [Internet]. [updated December 2016; cited January 2017]. http:// apps.who.int/healthinfo/statistics/mortality/whodpms/. Accessed 16 Jan 2018.

42. Allemani C, Matsuda T, Di Carlo V, Harewood R, Matz M, Nikšić M, et al. Global surveillance of trends in cancer survival 2000-14 (CONCORD-3): analysis of individual records for 37 513025 patients diagnosed with one of 18 cancers from 322 population-based registries in 71 countries. Lancet. 2018;391(10125):1023-75. 
43. Lara M, Gamboa C, Kahramanian MI, Morales LS, Hayes Bautista DE. Acculturation and Latino health in the United States: a review of the literature and its sociopolitical context. Annu Rev Public Health. 2005;26:367-97.

44. Cattin LM, Pinheiro PS, Callahan KE, Hage R. Twenty-first century cancer patterns in small island nations: Grenada and the English-speaking Caribbean. Cancer Causes Control. 2017;28(11):1241-9.

45. Gonzalez-Barrera A, Lopez MH. A demographic portrait of Mexican-origin Hispanics in the United States. Washington, DC: Pew Hispanic Center; 2013.

46. Boscoe FP, Henry KA, Sherman RL, Johnson CJ. The relationship between cancer incidence, stage and poverty in the United States. Int J Cancer. 2016;139(3):607-12.

47. Makarova Rusher OV, Altekruse SF, McNeel TS, Ulahannan S, Duffy AG, Graubard BI, et al. Population attributable fractions of risk factors for hepatocellular carcinoma in the United States. Cancer. 2016;122(11):1757-65.

48. Lavanchy D. Hepatitis B virus epidemiology, disease burden, treatment, and current and emerging prevention and control measures. J Viral Hepat. 2004;11(2):97-107.

49. Centers for Disease Control and Prevention (CDC). MMWR: recommendations for the identification of chronic hepatitis C virus infection among persons born during 1945-1965. United States: US Department of Health and Human Services; 2012. Report no.: 61(No. RR-4).

50. Sakala L. Breaking down mass incarceration in the 2010 census: state-by-state incarceration rates by race/ethnicity. Prison Policy Initiative; 2014.

51. Larney S, Kopinski H, Beckwith CG, Zaller ND, Jarlais DD, Hagan H, Rich JD, Bergh BJ, Degenhardt L. Incidence and prevalence of hepatitis $\mathrm{C}$ in prisons and other closed settings: results of a systematic review and meta-analysis. Hepatology. 2013;58(4):1215-24.

52. Kuniholm MH, Jung M, Everhart JE, Cotler S, Heiss G, McQuillan G, et al. Prevalence of hepatitis C virus infection in US Hispanic/Latino adults: results from the NHANES 2007-2010 and HCHS/SOL studies. J Infect Dis. 2014;209(10):1585-90.

53. US Dept. of Health and Human Services. National viral hepatitis action plan 2017-2020. 2017. https://www.hhs.gov/hepatitis/action-plan/national-viral-hepatitis-action-plan-overview/ index.html. Accessed 4 Feb 2018.

Open Access This chapter is licensed under the terms of the Creative Commons AttributionNonCommercial 4.0 International License (http://creativecommons.org/licenses/by-nc/4.0/), which permits any noncommercial use, sharing, adaptation, distribution and reproduction in any medium or format, as long as you give appropriate credit to the original author(s) and the source, provide a link to the Creative Commons license and indicate if changes were made.

The images or other third party material in this chapter are included in the chapter's Creative Commons license, unless indicated otherwise in a credit line to the material. If material is not included in the chapter's Creative Commons license and your intended use is not permitted by statutory regulation or exceeds the permitted use, you will need to obtain permission directly from the copyright holder. 